\title{
OCORRENNCIA DE AFLATOXINA EM FARELOS DE AMENDOIM (Arachis hypogaea L.) NA REGIÃO SOROCABANA DO ESTADO DE SÃO PAULO *
}

\author{
Homero Fonseca
}

\begin{abstract}
RESUMO
No presente trabalho foi estudada a ocorrência das aflatoxinas B e G em 44 amostras de farelo de amendoim provenientes de 6 fábricas da região Sorocabana, bem como uma possivel correlação na produção de ambas.

As amostras foram coletadas em quatro épocas representando material proveniente da industrialização de duas safras distintas a saber: março e maio - safra das «águas» e julho e setembro - safra da «seca».

Dos resultados concluiu-se que: a) a incidência de aflatoxina foi geral na região, pois todas as amostras estavam tóxicas; b) o nivel de toxidez encontrado foi elevado (valores de 0,1 a $10,0 \mathrm{ppm}$ ) sendo mais elevado na safra das «águas» - média de 3,78 ppm, contra 1,74 ppm na «seca»; c) apenas $4,54 \%$ do material examinado estaria em condições de ser utilizado para rações animais; d) praticamente não houve correlação entre a produção das aflatoxinas $B$ e $G$.
\end{abstract}

\section{INTRODUÇÃO}

Em 1960 uma misteriosa e aparentemente nova doença apareceu nas granjas da Inglaterra matando, em poucos meses, cerca de 100.000 peruzinhos. As aves morriam geralmente dentro de uma semana sendo, seus sintomas, a perda de apetite, diminuição da mobilidade, fraqueza das asas, das pernas, etc. A necrópsia sempre revela lesões necróticas no fígado e congestinamento nos rins (STEVENS ef al., 1960). Os autores, não conseguindo isolar nenhum agente infeccioso, suspeitaram que ela deveria ser de origem nutricional visto que, com a mudança da ração, frequentemente cessava a mortalidade, o que foi comprovado por SMITH (1960).

Logo em seguida vários pesquisadores descreveram a doença, dentre eles BLOUNT (1961) que a batizou de doença «X» dos perús.

Estudos posteriores revelaram que ela não era contagiosa e sim, era devida a material tóxico presentes nas rações, sendo fator comum do problema, uma partida de farelo de amendoim (ASPLIN \& CARNAGHAN, 1961).

* Entregue para publícação em 28/12/1973.

** Professor de Disciplina, Departamento de Tecnologia Rural da ESALQ. 
Isolado o princípio tóxico, verificou-se que ele era composto de quatro substâncias: $B_{1}, B_{2}, G_{1}$ e $G_{2}$. A estas substâncias foi dado nome genérico de aflatoxina pois, verificou-se que eram metabólitos produzidos pelo fungo Aspergillus flavus Link (SARGANT et al. 1961) que se desenvolvia sobre o amendoim, após a colheita, sob condições favoráveis de umidade do amendoim e de temperatura e umidade relativa do ar. Hoje são conhecidos outros metabólitos integrantes do grupo da aflatoxina, ou sejam, a $\mathrm{M}_{1}$ e $\mathrm{M}_{2}$ (De IONGH et al., 1964 e ALLCROFT et al. 1966) e que são as $B_{1}$ e $B_{2}$ metabolizadas e encontrada no leite e urina de vacas e ovelhas alimentadas com farelos tóxicos, e também são produzidas pelo próprio fungo. Além destas foram posteriormente descobertas as B2a, G2a (DUTTON \& HEATHCOTE, 1966, e as B3 e GM1 (DUTTON \& HEATHCOTE, 1969).

As propriedades carcinogênicas da aflatoxina foram constatadas em trutas (ASHLEY et al., 1964), ratos (WOGAN, 1965) e marrecos (SCHOENTAL, 1967).

Com relação ao homem os primeiros indícios de sua ação tóxica foram constatados por ZUCKERMAN \& FULTON (1966).

A aflatoxina é produzida pelo A .flavus e outros fungos, em quase todo mundo. RICHMOND et al. (1962a e 1962b) demonstraram que os efeitos tóxicos se reproduziam em marrecos, quando alimentados com soja, feijão («runner bean»), semente de algodão e trigo, sobre os quais tinha crescido o A. flavus.

A importância do problema tem despertado o interesse de governos e cientistas, no sentido de conhecerem a situação da aflatoxina em seus países, quer no amendoim, quer nas tortas e farelos. Já em 1961, na Nigéria, McDONALD \& HARKNESS (1963) fizeram uma investigação da presença de aflatoxina nas colheitas de amendoim provenientes de duas regiões distintas: $\mathrm{Ri}$ verain e do Norte. Das 40 amostras analisadas muitas se apresentaram isentas da toxina, outras com valores até $0,5 \mathrm{ppm}$, apenas 3 ultrapassando este limite.

SELLSCHOP ef al., (1965) relatam um levantamento feito na África do Sul por uma equipe de técnicos nos anos de 1963 e 1964, quando examinaram a ocorrência de aflatoxina em cerca de 2.000 amostras de amendoim e sub-produtos provenientes das províncias Transvaal, Natal e Cabo e do Estado Livre de Orange. As amostras analisadas no primeiro ano mostraram toxidez bastante elevada tendo, algumas partidas, apresentado $75 \%$ das amostras com mais de $2,0 \mathrm{ppm}$. Já no ano seguinte, o número de amostras tóxicas bem como o nível de toxidez decresceu o que, segundo aqueles autores, deve ter sido em virtude de medidas restritivas na aceitação de amendoim mofado.

PEERS (1965) apresentou uma das melhores pesquisas efetuadas neste campo, ao relatar o trabalho por ele feito em Vom, no Norte da Nigéria, durante os anos de 1963, 1964 e 1965, em dez apartidas de amendoim provenientes da região de Zaria. Seu trabalho visou o controle da aflatoxina em tortas e farelos, resultantes daquele amendoim, e destinados à composição do Arlac*, bem como verificar o efeito das medidas de melhoria das condi- 
ções de secagem armazenamento e seleção do amendoim. Estes estudos revelaram que a toxidez das primeiras partidas de torta, em 1963 foi em média de $0,34 \mathrm{ppm}$ e que, com as melhorias introduzidas na secagem e armazenamento, a toxidez foi baixando até estabilizar-se, nas duas últimas partidas de 1965, em cerca de 0,02 ppm.

CROWTER (1966), no período de fins de 1965 a meados de 1966, examinou 186 amostras de amendoim e farelos de amendoim provenientes de várias regiões, em Gâmbia, cujos resultados variaram bastante. $\mathrm{Na}$ primeira partida, 53\% das amostras eram de toxidez «Negativa», $19 \%$ «Média» e $28 \%$ «Alta», não se registrando nenhuma na categoria «Muito alta». Já em partida proveniente de outra região, constatou farelos de toxidez muito elevada chegando até $40 \mathrm{ppm}$ de B1 e $30 \mathrm{ppm}$ de G1.

Entre nós MENEZES et al. (1966), em trabalho que consideraram preliminar, determinaram aflatoxina em amendoim, tortas e farelos procedentes de várias fábricas do Estado de São Paulo. Mais de $90 \%$ delas apresentaram teores elevados de aflatoxina e consideraram que apenas $3 \%$ das tortas e farelos estariam em condições de serem utilizados em rações para animais.

TANGO ef al. (1967), trabalhando com amendoim das «águas» e da «seca», investigaram principalmente, a incidência de aflatoxina e o grau de infestação de fungos. Os resultados mostraram uma maior incidência de aflatoxina na safra das «águas» com $59 \%$ das amostras nas categorias «Alta» e «Muito Alta». Inversamente, encontraram um maior grau de infestação de fungos, na safra da «seca».

Sendo a aflatoxina um problema de grande importância, que pode atingir o homem não só diretamente, pelo consumo de produtos contendo amendoim contaminado, como também indiretamente, através de leite produzido por animais que eventualmente se alimentem de farelos tóxicos, resolvemos investigar sua ocorrência em nosso Estado, através do farelo de amendoim, subproduto da indústria de extração de óleo. Nela procurou-se esclarecer principalmente, os seguintes pontos: a) nível de toxidez dos farelos; b) variação da toxidez entre épocas de coleta das amostras e c) possível correlação entre a produção das aflatoxinas $\mathrm{B}$ e $\mathrm{G}$.

Incluiu-se este último ítem por nada termos encontrado na literatura, com relação a ele, em trabalhos desta natureza.

\section{MATERIAL E MÉTODOS}

\section{Material}

O material utilizado para execucão deste trabalho constou de 44 amostras de farelo de amendoim da safra de 1966/67 coletadas em seis fábricas de óleo,

(*) Concentradn nroteico destinado à infância no qual o farelo de amendoin entrava na nroporção de $33 \%$. 
localizadas na região servida pela Estrada de Ferro Sorocabana, compreendida entre Ourinhos e Santo Anastácio, no Estado de São Paulo.

O cultivo do amendoim, que é feito em duas épocas do ano, permite a obtenção de duas colheitas por ano, que se processam na estação chuvosa, por volta de janeiro, e na estação seca, por volta de junho, e por essa razão, são chamados, respectivamente de amendoim das «águas» e da «seca».

As amostras foram coletadas em quatro épocas, a saber: março e maio, representando material proveniente da industrialização da safra das «águas», num total de 24 amostras e julho e setembro, representando material da safra da «seca», num total de 20 amostras, visto que, nesta safra, apenas cinco fábricas trabalharam.

De cada fábrica foi retirada uma amostra de cerca de $1 \mathrm{~kg}$, representativa de todo o material existente na fábrica e recolhida em sacos plásticos o qual era, posteriormente, colocado em sacos de papel, sobre os quais era feita a identificação da fábrica e da época da colheita.

As fábricas foram identificadas por números e as épocas de coleta com os nomes dos meses em que foram retiradas as amostras. letas.

As análises foram efetuadas nos dias imediatos após o término das co-

\section{Métodos}

\section{Preparo das amostras}

As amostras foram trituradas passando-se quatro a cinco vezes em moinho de discos («Disc Mill», modelo 4E, The Straub Co., Philadelphia, EE.UU.) para obtenção da finura adequada para a análise sendo, posteriormente, passadas em peneiras de crivo de 841 micra (20 mesh»). Em seguida as amostras foram divididas em duas sub amostras, a e b, que foram novamente guardadas em sacos plásticos, até o momento de serem analisadas.

\section{Extração da toxina}

De cada sub-amostra, daqui para frente denominadas simplesmente de amostra, foram tomados $20 \mathrm{~g}$, dos quais foi extraida a toxina com clorofórmio, de acordo com o método de LEE (1965). O filtro obtido foi denominado de solução $X$. Desta foram tomados $8 \mathrm{ml}$ e diluidos a $100 \mathrm{ml}$ com clorofórmio: solução $\mathbf{Y}$.

\section{Preparo das cromotoplacas}

Placas de vidro de $10 \times 20 \mathrm{~cm}$ com camada de $500 \mu$ de silicagel-G, foram preparadas segundo a técnica de COOMES \& FEUELL (1965).

\section{Cromatografia do extrato}

A dois $\mathrm{cm}$ da base das cromatoplacas foram colocadas as seguintes alíquotas: $20 \mu l$ dạ solução $X$ e $25,10,5$ e 2,5 $\mu$ l da solução $Y$, além de uma alí- 
quota de concentração conhecida, para comparação, e desenvolvidas com o solvente clorofórmio-metanol $95: 5$, até $10 \mathrm{~cm}$ acima do local das amostras, em câmaras saturadas.

\section{Deferminação do teor de aflatoxina e sua correspondente toxidez.}

As placas, após a cromatografia, foram examinadas, em sala escura, a distância de $30 \mathrm{~cm}$ de uma lâmpada ultra-violeta Philips, tipo HPW, 125 watt, com emissão máxima em $365 \mathrm{~nm}$ e observada a presença ou ausência de manchas fluorescentes, azul-violeta das aflatoxinas B1 $+\mathrm{B} 2$, num $\mathrm{Rf}=$ $0,50-0,55$ e esverdeada das aflatoxinas $\mathrm{G} 1+\mathrm{G} 2$, num $\mathrm{Rf}=0,45-0,50$.

O cálculo da concentração foi efetuado segundo COOMES \& FEUELL (1965). Este sistema forneceu resultados entre dois limites de concentração. Assim, a menor alíquota em que foi observada fluorescência, nos forneceu o limite inferior. Para o limite superior foi tomada a alíquota seguinte em que não foi observada fluorescência. Nos casos em que foi observada fluorescência na menor das alíquotas, a solução $\mathbf{Y}$ foi rediluida e recromatografada para verificarmos o limite superior. Estes limites, em função da menor alíquota foi observada fluorescência, foram:

\begin{tabular}{|c|c|c|c|c|c|c|c|c|}
\hline \multicolumn{3}{|c|}{ Alíquota } & B1 & + & B2 & G1 & & G2 \\
\hline 20 & HIsol. $\mathrm{X}$ & - & 0,10 & - & 1,00 & 0,075 & - & 0,75 \\
\hline 5 & $\mu l$ sol. $Y$ & - & 1,00 & - & 2,50 & 0,75 & - & 1,87 \\
\hline 0 & $\mu l$ sol. Y & - & 2,50 & - & 5,00 & 1,87 & - & 3,75 \\
\hline 5 & $\mu l$ sol. $Y$ & - & 5,00 & - & 10,00 & 3,75 & - & 7,50 \\
\hline 2,5 & $\mu l$ sol. $Y$ & - & 10,00 & - & 20,00 & 7,50 & - & 15,00 \\
\hline
\end{tabular}

Para a avaliação da toxidez das amostras os resultados foram enquadrados nas categorias de toxidez estabelecidas pelo TROPICAL PRODUCTS INSTTTUTE (1962) e que consta do QUADRO 1.

Quadro 1 - Relação entre a concentração de aflatoxina $B_{1}$ e toxidez do material.

\begin{tabular}{ll}
\hline Categoria de toxidez & Nivel de aflatoxina $\mathrm{B}_{1}$ \\
\hline Muito Alta & Acima de $1,00 \mathrm{ppm}$ \\
Alta & Entre 0,25 e $1,00 \mathrm{ppm}$ \\
Média & Entre 0,05 e $0,25 \mathrm{ppm}$ \\
Baixa ou Negativa & Abaixo de $0,05 \mathrm{ppm}$ \\
\hline
\end{tabular}


A nossa escala de categoria foi ligeiramente modificada, conforme pode ser observado, para maior facilidade dos trabalhos de análise. Além disso, quando do início dos nossos trabalhos, verificamos que os valores encontrados eram bastante elevados e por isso resolvemos esmiuçar mais a categoria «Muito Alta», ou seja, farelos com mais de $1,00 \mathrm{ppm}$. Por esse motivo subdividimo-la em quatro níveis.

\section{Análise estatistica}

Para a computação de médias foi usado o centro dos intervalos dos níveis (PEERS, 1965) com as variáveis transformadas em $\log (x+1)$ de acordo com SNEDECOR (1956) e STEEL \& TORRIE (1960).

$O$ cálculo da correlação entre as aflatoxinas B e $\mathrm{G}$ foi feito conforme SNEDECOR (1956), também com as variáveis transformadas em $\log (x+1)$.

Para a análise da variância dos dados foi considerado o modelo:

$$
\mathrm{Y}=\mathrm{u}+\mathrm{fi}+\mathrm{sj}+(\mathrm{fs})_{\mathrm{ij}}+\mathrm{m}_{\mathrm{k}(\mathrm{j})}+(\mathrm{fm})_{\mathrm{ik}(\mathrm{j})}+\mathrm{a}_{1(\mathrm{ijk})}
$$

onde: $\quad i=1,2,3, \ldots \ldots \ldots \ldots . .6$

$$
\begin{aligned}
& \mathrm{j}=1,2 . \\
& \mathrm{r}=1,2 . \\
& \mathrm{l}=1,2 .
\end{aligned}
$$

tendo os efeitos, todos aleatórios, o seguinte significado:

$$
\begin{aligned}
\mathrm{f} & =\text { efeitos de fábrica } \\
\mathrm{s} & =\text { efeito de safras } \\
(\mathrm{fs}) & =\text { efeito da interação fábrica } \mathrm{x} \text { safra } \\
\mathrm{m} & =\text { efeito do mês (ou época) dentro da safra } \\
(\mathrm{fm}) & =\text { efeito da interação fábrica x mes, dentro da safra } \\
\mathrm{a} & =\text { efeito da amostra. }
\end{aligned}
$$

A análise da variância foi feita para estimar as variâncias relativas a cada efeito. Os dados também foram transformados em $\log (x+1)$.

A esperança matemática dos quadrados médios foi computada conforme BENNETT \& FRANKLIN (1954).

Os centros dos intervalos dos níveis com os quais foram feitos os calculos acima referidos, foram os seguintes: 


$\begin{array}{rrllll} & \text { Intervalo } & \text { Centro } & & \text { Intervalo } & \text { Centro } \\ 0,00 & -0,05 & 0,025 & 0,00 & -0,075 & 0,0375 \\ 0,05 & -0,10 & 0,075 & 0,075 & -0,75 & 0,4125 \\ 0,10-1,00 & 0,55 & 0,75 & -1,87 & 1,31 \\ 1,00 & -2,50 & 1,75 & 1,87 & -3,75 & 2,81 \\ 2,50 & -5,00 & 3,75 & 3,75 & -7,50 & 5,625 \\ 5,00 & -10,00 & 7,50 & 7,50 & -15,00 & 11,25 \\ 10,00-20,00 & 15,00 & & - & \end{array}$

\section{RESULTADOS E DISCUSSÃO}

\section{Resultados}

Os resultados gerais estão nos quadros de números 2 a 15.

\section{Discussão}

Neste trabalho os resultados são apresentados em níveis de toxidez, pois pela sua natureza, um levantamento, desejou-se saber apenas a extensão e o nível de incidência da toxina. Este procedimento é corrente entre os autores, como pode ser observado em PEERS (1965), SELLSCHOP et al. (1965), MENEZES et al. (1966) e TANGO et al. (1967).

Os níveis de toxidez estão intimamente relacionados com os testes biológicos em marrequinhos de um dia, pois é evidente que os números absolutos do conteúdo de aflatoxina não darão qualquer informação de sua toxidez a não ser quando relacionados com os seus efeitos nos referidos testes.

Conforme pode-se observar nos quadros de números 2 a 5 , a aflatoxina esteve presente $\mathrm{m}$ todas as amostras e variou entre os limites de 0,1 e 10,0 ppm para as aflatoxinas B e entre 0,075 e 3,75 ppm para as $\mathrm{G}$.

Baseados nos resultados apresentados para as aflatoxinas $\mathrm{B}$ podemos verificar pelo Quadro 11, que não temos nenhuma incidência nas categorias «Baixa ou Negativa» e «Média», que $4,54 \%$ estão na categoria «Alta» e que 95,46\% estão na «Muito Alta».

A toxidez dos farelos como se vê, foi bastante elevada. Para que se tenha uma idéia do significado dos resultados encontrados podemos citar que a concentração de aflatoxina B1 no farelo que dizimou os peruzinhos na Inglaterra em 1960 estava entre 2,0 e 5,0 ppm. Como pode-se observar, ainda com dados do Quadro 11, 49,99\% das amostras estavam com mais de 2,5 ppm.

Estes resultados estão de acordo com os encontrados entre nós por MENEZES ef al. (1966) e com os de TANGO ef al. (1967).

Considerando-se recomendações de instituições que pesquisaram extensivamente o assunto, (ANONIMO, 1969) pelas quais os farelos ou tortas com 
mais de 1,00 ppm praticamente não podem ser utilizados em rações animais em nenhuma proporção, não poderiamos aproveitar mais que $4,54 \%$ do material examinado.

A situação em outros países é bastante variável conforme pode-se observar em McDONALD \& HARKNESS (1963) na Nigéria, SELLSCHOP ef al. (1965) na África do Sul, PEERS (1965) em Vom, também na Nigéria, LIM \& YEAP (1966) na Malásia e CROWTHER (1966) em Gâmbia. Todavia pode-se constatar facilmente que o nível de toxidez dos farelos por nós examinados foi bem mais elevado que o encontrado naqueles países.

Pela análise da variância dos resultados podemos verificar que não houve diferença de comportamento entre as fábricas, tanto para as aflatoxinas B como para as G. Houve influência da safra, significativa ao nível de 5\% de probabilidade, apenas com relação às aflatoxinas B. Este comportamento pode ser constatado através dos quadros números 13 e 14 .

Note-se que as médias encontradas no Quadro 15, também espelham essa característica.

Quadro 15 - Médias dos valores encontrados para as aflatoxinas B e G, por safra e geral. (expressas em partes por mullão)

\begin{tabular}{cccc}
\hline Aflatoxına & "Águas" & "Seca" & Geral \\
\hline B & 3.78 & 1,74 & 2,62 \\
G & 0,66 & 0,76 & 0,71 \\
\hline
\end{tabular}

A época ou mês de coleta dentro de uma mesma safra também não variou significativamente, assim com a interação fábrica vs safra. Houve interação mês vs fábrica dentro da safra porém apenas a aflatoxina $B$.

Pelos quadros de números 8 e 9 pode-se verificar que, com relação à aflatoxina B, o maior fator de variação foi a safra com $66,39 \%$ do total, ao passo que para a aflatoxina $G$ o maior fator de variação foi a amostragem. A variação dos demais fatores foi pequena.

Esse comportamento da aflatoxina B já era esperado pois a safra das «águas» geralmente apresenta condições mais propícias para o desenvolvivimento de fungos que a safra da «seca». Tanto o valor encontrado para a safra da «águas» como o da «seca» foram elevados.

Todos os farelos examinados eram tóxicos e apresentaram ambas as aflatoxinas. Os valores encontrados para as aflatoxinas B foram sempre mais elevados que os da G numa mesma amostra.

Ocorreu-nos pois investigar também se havia correlação entre a produção dos metabólitos, visto que, em trabalhos de campo, não há referência na literatura sobre o assunto. $O$ resultado obtido no estudo dessa possível 
correlação foi de $\mathbf{r}=0,39$ (QUADRO 10) não significativo podendo-se considerar que praticamente não houve correlação.

\section{CONCLUSOES}

Dos resultados obtidos no presente trabalho pode-se tirar as seguintes conclusões:

1) A incidência da aflatoxina foi geral na região Sorocabana, pois todas as amostras eram tóxicas.

2) O nível de toxidez foi, no geral, muito elevado com algumas amostras chegando a $10,0 \mathrm{ppm}$.

3) Os farelos da safra das «águas» apresentaram maior toxidez (média de $3,78 \mathrm{ppm}$ ) que os da «seca» (média de 1,74 ppm)

4) Apenas 4,54\% do material analisado poderia ser aproveitado para rações animais pois $95,46 \%$ apresentou níveis de aflatoxina que o enquadrou na categoria «Muito Alta».

5) Praticamente não houve correlação entre a produção das aflatoxinas B e G.

\section{SUMMARY}

OCCURRENCE OF AFLATOXIN IN PEANUT FLOUR IN THE REGION SOROCABANA, IN THE STATE OF SÃO PAULO.

In the present work, the occurrence of the aflatoxins $B$ and $G$, as well as a possible correlation between both were studied in 44 samples of peanut flour from 6 oil mills of the region Sorocabana, in the State of São Paulo.

The samples were obtained in four collections representing material from two crops in two different seasons: March and May, in the rainy season and July and September, in the dry season.

From the results it was concluded that: a) the occurrence of aflatoxin was generalized in that region, for all the samples were toxic; b) the toxicity level was found to be very high (figures from 0.1 to $10.0 \mathrm{ppm}$ ) being higher in the rainy season - average of $3.78 \mathrm{ppm}$, agains $1.74 \mathrm{ppm}$ in the dry season; c) it was considered that only $4.54 \%$ of the material examined could be utilized for admixture in feedstuffs; d) it was not found correlation in the production of the aflatoxins $B$ and $G$.

\section{LITERATURA CITADA}

ALLCROFT, R., H. ROGERS, G. LEWIS, J. NABNEY \& P. E. BEST, $1966-$ Metabolism of aflatoxin in sheep: excretion of the milk toxin. Nature, 209: 154-155.

ANONIMO, 1969 - Informationsdienst Futter und Fütterung. Ed. Fachverband der Futtermittelindustrie e. V., Hamburg. 
ASPLIN, F. D. \& R. B. A. CARNAGHAN, 1961 - The toxicity of certain groundnut meals for poultry with special reference to their effect on ducklings and chickens. Vet. Rec., 73: 1215-1219

BLOUNT, W. P., 1961 - Turkey " $X$ " disease. Turkeys, 9: 52, 55-58, 61, 77.

COOMES, T. J. \& A. L. FEUELL, 1965 - Recommended procedures tor the detection of aflatoxin $\mathrm{B}_{1}$ in grondnuts and groundnut materials. Tropical Products Institute, Report $n .^{\circ}$ G 13, M. O. D., Londres.

CROWTHER, P. C., 1966 - Report of the Produce Chemist, $4^{\text {th }}$ november 1965 - $4^{\text {th }}$ may 1966. The Laboratory, Dept. of Agriculture, Yundum Experimental Station, The Gambia. 15 pp.

BENNETT, C. A. \& N. L. FRANKLIN, 1963 - Statistical Analysis in Chemistry and the Chemical Industry. John Wiley \& Sons, Inc., N. York, 724 pp.

De IONGH, H., R. O. VLES \& J. G. Van PELT, 1964 - Milk of mammals fed an aflatoxin - containing diet. Nature, 202: 466-467.

DUTTON, M. F. \& J. G. HEATHCOTE, 1966 - Two new aflatoxins. Biochem. J., 101: 21P-22P.

DUTTON, M. F. \& J. G. HEATHCOTE, 1969 - Some interesting relationship between the new aflatoxins and their associated metabolites. The J. South African Chem. Inst. $X X I I: 5107-5118$.

LEE, W. V., 1965 - Quantitative determination of aflatoxin in groundnut products. Analyst (Lond.) 90 (1070) : 305-307.

MCDONALD, D. \& C. HARKNESS, 1963 - Growth of Aspergillus flavus and production of aflatoxin in groundnuts. Part II. Trop. Sci., 53 (3) : 143-154.

MENEZES, T. J. B., J. S. TANGO, F. A. S. COELHO \& C. G. TEIXEIRA, 1966 - Ocorrência do Aspergillus flavus e da aflatoxina em sementes e farelo de amendoim. Apresentado à XVIII Reunião Anual da S. B. P. C., Blumenau, S.C., 1966.

PEERS, F. G., 1965 - Summary of the work done at Vom (Northern Nigeria) on Aflatoxin levels in grondnut flour and Arlac. Nutr. Docum. Aflatoxin/8. WHO/FAO/UNICEF. P. A. G. 1965 Meeting, Rome.

RICHMOND , J. W., N. H. SUTCLIFFE, N. W. R. DANIELS, P. W. RUSSELEGGITT \& J. B. M. COPPOCK, 1962a - Factors other than groundnut relating to "turkey" X disease". Vet. Rec., 74 (18) : 544-545.

RICHMOND, J. W., N. H. SUTCLIFFE, N. W. R. DANIELS, P. W. RUSSELEGGIT \& J. B. COPPOCK, 1962b - Factors other than groundnut in the production of aflatoxin. Vet. Rec. 74 (35) 962.

SARGEANT, K., A. SHERIDAN, J. O'KELLY \& R. B. A. CARNAGHAN, 1961 - Toxicity associated with certain samples of groundnuts. Nature, 192: 10961097.

SCHOENTAL, R., 1967 - Aflatoxins. In; "Annual Review of Pharmacology" 7: 343-356.

SELLSCHOP, J. P. F., N. J. KRIEK \& J. C. G. du PREEZ, 1965 - Distribution and degree of occurrence of aflatoxin in groundnuts and groundnut products. Symp. Mycotoxins Foodstuffs, Agric. Aspects. Febr. 1965, Pretoria, South Africa : 9-17.

SMITH, K. M., 1960 - Disease of turkey poults. Vet. Rec. 72 (32) : 652.

SNEDECOR, C. W., 1956 - Statistical Methods. The Iowa State College Press, Ames, Iowa, EUA., $5^{\mathrm{a}} \mathrm{Ed} .534 \mathrm{pp}$.

STEEL, R. G. D. \& J. H. TORRIE, 1960 - Principles and Procedures of Statistics. McGraw-Hill Book Co., Inc., N. York, 481 pp. 
STEVENS, A. J., C. N. SANDERS, J. B. SPENCE \& A. G. NEWHAM, $1960-$ Investigations into "diseases" of turkey poults. Vet. Rec., 72 (31) 627.

TANGO, S. J., T. J. B. MENEZES \& C. G. TEIXEIRA, 1967 -- Levantamento da ocorrência da aflatoxina em sementes de amendoim nas safras das águas e da seca de 1965. Apresentado à XIX Reunião Anual da S. B. P. C., Rio de Janeiro, 1967.

TROPICAL PRODUCTS INTITUTE, 1962 - Aflatoxin in groundnuts and groundnut products. Interpretation of physico-chemical and biological test results. T. P. I., Ministry of Overseas Development, Londres, $1 \mathrm{p}$.

WOGAN, G. N., 1965 - Experimental toxicity and carcinogenicity of aflatoxins. In "Mycotoxins in Foodstuffs". Ed. G. N. Wogan, The M. I. T. Press, Cambridge, Mass. EE.UU.

ZUCKERMAN, A. J. \& F. FULTON, 1966 - Acute toxic effects of on human embryo liver cell in culture Brit. Med. J., 2: 90-91.

\section{AGRADECIMENTOS}

Agradecemos à FAPESP pelo auxílio financeiro concedido para a realização desta pesquisa e à UNICEF pelo auxílio em drogas e aparelhos. Agradecemos também ao Prof. Roland Vencovsky e ao acadêmico de agronomia Isaias 0 . Geraldi pela execução das análises estatísticas. 
Quadro 2 - Ocorrência das aflatoxinas B e G em amostras da safra das "águas" MARÇO (expressa em ppm)

\begin{tabular}{|c|c|c|c|c|c|c|c|c|}
\hline \multirow{2}{*}{$\begin{array}{l}\text { Fábrica } \\
\text { número }\end{array}$} & \multicolumn{4}{|c|}{ Amostra a } & \multicolumn{4}{|c|}{ Amostra $b$} \\
\hline & & B & $\mathrm{C}$ & & & B & & $\mathrm{G}$ \\
\hline 1 & 2,5 & $-5,0$ & 0,075 & $-0,75$ & 2,5 & - & 5,0 & $0,75-1,87$ \\
\hline 2 & 1,0 & $-2,5$ & 0,075 & $-0,75$ & 1,0 & - & 2,5 & $0 ; 075-0,75$ \\
\hline 3 & 2,5 & $-5,0$ & 0,75 & $-1,87$ & 2,5 & - & 5,0 & $0,75-1,87$ \\
\hline 4 & 2,5 & $-5,0$ & 0,75 & $-1,87$ & 2,5 & - & 5,0 & $0,075-0,75$ \\
\hline 5 & 1,0 & $-2,5$ & 0,075 & $-0,75$ & 1,0 & - & 2,5 & $0,075-0,75$ \\
\hline 6 & 2,5 & $-5,0$ & 0,075 & $-0,75$ & 5,0 & - & 10,0 & $0,75-1,87$ \\
\hline
\end{tabular}

Quadro 3 - Ocorrência das aflatoxinas B e G em amostras da safra das "águas" MAIO (expressa em ppm).

\begin{tabular}{|c|c|c|c|c|c|c|c|c|}
\hline \multirow{2}{*}{$\begin{array}{l}\text { Fábrica } \\
\text { número }\end{array}$} & \multicolumn{4}{|c|}{ Amostra a } & \multicolumn{4}{|c|}{ Amostra b } \\
\hline & & B & & G & & B & & G \\
\hline 1 & 2,5 & $-5,0$ & 0,075 & $-0,75$ & 2,5 & - & 5,0 & $0,075-0,75$ \\
\hline 2 & 5,0 & $-10,0$ & 0,075 & $-0,75$ & 5,0 & - & 10,0 & $0,075-0,75$ \\
\hline 3 & 2,5 & $-5,0$ & 0,75 & $-1,87$ & 5,0 & - & 10,0 & $0,075-0,75$ \\
\hline 4 & 2,5 & $-5,0$ & 0,075 & $-0,75$ & 2,5 & - & 5,0 & $0,075-0,75$ \\
\hline 5 & 2,5 & $-5,0$ & 0,075 & $-0,75$ & 2,5 & - & 5,0 & $0,075-0,75$ \\
\hline 6 & 2,5 & $-5,0$ & 0,75 & $-1,87$ & 2,5 & - & 5,0 & $0,75-1,87$ \\
\hline
\end{tabular}


Quadro 4 - Ocorrência das aflatoxinas B e G em amostras da safra da "seca" JULHO.

\begin{tabular}{|c|c|c|c|c|c|c|c|c|}
\hline \multirow{2}{*}{$\begin{array}{l}\text { Fábrica } \\
\text { número }\end{array}$} & \multicolumn{4}{|c|}{ Amostra a } & \multicolumn{4}{|c|}{ Amostra $b$} \\
\hline & & B & & G & & B & & G \\
\hline 1 & 1,0 & $-2,5$ & 0,075 & $-0,75$ & 1,0 & - & 2,5 & $0,75-1,87$ \\
\hline 3 & 1,0 & $-2,5$ & 0,075 & $-0,75$ & 1,0 & - & 2,5 & $0,075-0,75$ \\
\hline 4 & 2,5 & $-5,0$ & 0,075 & $-0,75$ & 1,0 & - & 2,5 & $0,075-0,75$ \\
\hline 5 & 1,0 & $-2,5$ & 0,075 & $-0,75$ & 1,0 & - & 2,5 & $0,75-1,87$ \\
\hline 6 & 2,5 & $-5,0$ & 1,87 & $-3,75$ & 1,0 & - & 2,5 & $0,75-1,87$ \\
\hline
\end{tabular}

Obs.: A fábrica de no 2 não trabalhou com a safra da "seca".

Quadro 5 - Ocorrência das aflatoxinas B e G em amostras da safra da "seca" SETEMBRO.

\begin{tabular}{|c|c|c|c|c|c|c|c|c|}
\hline \multirow{2}{*}{$\begin{array}{l}\text { Fábrica } \\
\text { número }\end{array}$} & \multicolumn{4}{|c|}{ Amostra a } & \multicolumn{4}{|c|}{ Amostra $b$} \\
\hline & & B & & G & & B & & $\mathbf{G}$ \\
\hline 1 & 0,1 & $-1,0$ & 0,075 & $-0,75$ & 0,1 & - & 1,0 & $0,075-0,75$ \\
\hline 3 & 1,0 & $-2,5$ & 0,075 & $-0,75$ & 1,0 & - & 2,5 & $0,075-0,75$ \\
\hline 4 & 1,0 & $-2,5$ & 0,75 & $-1,87$ & 1,0 & - & 2,5 & $0,75-1,87$ \\
\hline 5 & 1,0 & $-2,5$ & 0,075 & $-0,75$ & 1,0 & - & 2,5 & $0,075-0,75$ \\
\hline 6 & 1,0 & $-2,5$ & 0,75 & $-1,87$ & 1,0 & - & 2,5 & $0,75-1,87$ \\
\hline
\end{tabular}

Obs.: A fábrica de no 2 não trabalhou com a safra da "seca". 
Quadro 6 - Análise de variância das aflatoxinas B.

\begin{tabular}{lcccc}
\hline Causa de variação & G.L. & S.Q. & Q.M. & F. \\
\hline Fábrica (F) & 4 & 0,104230 & 0,026065 & 1,41 n.s. \\
Safras (S) & 1 & 0,576432 & 0,576432 & $12,40^{*}$ \\
Interação F x S & 4 & 0,074164 & 0,018541 & 1,24 n.s. \\
Mes da safra (M) & 2 & 0,058589 & 0,029295 & 1,95 n.s. \\
M x F dentro de S & 8 & 0,119880 & 0,014985 & $2,49 *$ \\
Amostragem & 20 & 0,120207 & 0,006010 & \\
\hline T O T A L & 39 & 1,053502 & & \\
\hline
\end{tabular}

Quadro 7 - Análise da variância das aflatoxinas G.

\begin{tabular}{lcccc}
\hline Causa de Variação & G.L. & S.Q. & Q.M. & F. \\
\hline Fábricas (F) & 4 & 0,168497 & 0,042124 & 1,97 n.s. \\
Safras (S) & 1 & 0,001181 & 0,001181 & 0,41 n.s. \\
Interação F x S & 4 & 0,085319 & 0,021330 & 1,68 n.s. \\
Mes de safra (M) & 2 & 0,025221 & 0,012611 & 0,99 n.s. \\
M x F dentro de S & 8 & 0,101506 & 0,012688 & 1,57 n.s. \\
Amostragem & 20 & 0,161518 & 0,008076 & \\
\hline T O T A L & 39 & 0,543242 & & \\
\hline
\end{tabular}


Quadro 8 - Estimativa das variâncias de cada fator de variação para as aflatoxinas B.

\begin{tabular}{llrr}
\hline Fator de variação & \multicolumn{2}{c}{$\hat{\mathbf{G}}^{2}$} & $\%$ \\
\hline Fábricas (F) & $\boldsymbol{G}^{2} \mathrm{f}$ & 0,000940 & 2,30 \\
Safras (S) & $\boldsymbol{\sigma}^{2}$ & 0,027179 & 66,39 \\
F x S & $\boldsymbol{G}^{2}$ fs & 0,000889 & 2,17 \\
Meses d. safra (M) & $\sigma^{2} \mathrm{~m}$ & 0,001431 & 3,50 \\
M x F dentro de S & $\boldsymbol{G}_{\mathrm{mf}}^{2}$ & 0,004487 & 10,96 \\
Amostragem & $\sigma^{2}$ & 0,006010 & 14,68 \\
\hline T O T A L & & 0,040936 & 100,00 \\
\hline
\end{tabular}

Quadro 9 - Estimativa das variâncias de cada fator de variação para as aflatoxinas G.

\begin{tabular}{llrr}
\hline Fator de variação & \multicolumn{2}{c}{$\hat{G}^{2}$} & $\%$ \\
\hline Fábricas (F) & $G^{2} \mathrm{f}$ & 0,002599 & 17,16 \\
Safras (S) & $G^{2} \mathrm{~s}$ & $-0,001003$ & 0,00 \\
F x S & $G^{2} \mathrm{fs}$ & 0,002160 & 14,27 \\
Meses d. safra (M) & $\mathcal{G}^{2} \mathrm{~m}$ & $-0,000007$ & 0,00 \\
M x F dentro de S & $\mathrm{G}^{2} \mathrm{mf}$ & 0,002306 & 15,23 \\
Amostragem & $G^{2}$ & 0,008076 & 53,54 \\
\hline T O T A L & & 0,015141 & 100,00 \\
\hline
\end{tabular}

Quadro 10 - Correlação entre as aflatoxinas B e G.

$$
\overline{\mathrm{r}}=0,39 \text { n.s. }
$$


Quadro 11 - Distribuição do número de incidências das aflatoxinas $B$, por níveis e respectivas categorias de toxidez. (expressa em números absolutos ( $\mathrm{n}$ ) e percentagens).

\begin{tabular}{rrrrl}
\hline & Nivel & n & $\%$ & Categoria de toxidez \\
\hline $0,0-0,05$ & 0 & 0 & 0,00 & Baixa ou Negativa \\
$0,05-$ & $-0,1$ & 2 & 0,00 & Média \\
$0,1-$ & 1,0 & 4,54 & Alta \\
\hline $1,0-2,5$ & 20 & 45,46 & \\
$2,5-5,0$ & 18 & 40,91 & Muito alta \\
$5,0-10,0$ & 4 & 9,09 & \\
$10,0-20,0$ & 0 & 0,00 & \\
\hline T O T A L & & 44 & 100,00 & \\
\hline
\end{tabular}

Quadro 12 - Distribuição do número de incidências das aflatoxinas G, por níveis. (expressa em números absolutos ( $n$ ) e percentagens).

\begin{tabular}{cccc}
\hline Nivel & & $\mathrm{n}$ & $\%$ \\
\hline $0,00-0,075$ & 0 & 0,00 \\
$0,075-0,75$ & 28 & 63,63 \\
$0,75-1,87$ & 15 & 34,10 \\
$1,87-3,75$ & 1 & 2,27 \\
$3,75-7,50$ & 0 & 0,00 \\
$7,50-15,00$ & 0 & 0,00 \\
\hline T O T A L & & 44 & 100,00 \\
\hline
\end{tabular}


Quadro 13 - Distribuição do número de incidências das aflatoxinas B por níveis e por safras. (expressa em números absolutos (n) e percentagens).

\begin{tabular}{rrrrrr}
\hline & \multirow{2}{*}{ Nivel } & \multicolumn{2}{c}{ “Äguas" } & \multicolumn{3}{c}{ "Seca" } \\
\cline { 3 - 6 } & & $\mathrm{n}$ & $\%$ & $\mathrm{n}$ & $\%$ \\
\hline $0,0-0,05$ & 0 & 0,00 & 0 & 0,00 \\
$0,05-0,1$ & 0 & 0,00 & 0 & 0,00 \\
$0,1-1,0$ & 0 & 0,00 & 2 & 10,00 \\
$1,0-$ & 2,5 & 4 & 16,665 & 16 & 80,00 \\
$2,5-5,0$ & 16 & 66,67 & 2 & 10,00 \\
$5,0-10,0$ & 4 & 16,665 & 0 & 0,00 \\
$10,0-20,0$ & 0 & 0,00 & 0 & 0,00 \\
\hline T O T A L & 24 & 100,00 & 20 & 100,00 \\
\hline
\end{tabular}

Quadro 14 - Distribuição do número de incidências das aflatoxinas $G$, por níveis e por safras.

(expressa em números absolutos (n) e percentagens).

\begin{tabular}{lrrrrr}
\hline \multirow{2}{*}{ Nivel } & \multicolumn{3}{c}{ “Águas" } & \multicolumn{3}{c}{ "Seca" } \\
\cline { 2 - 6 } & & $\mathrm{n}$ & $\%$ & $\mathrm{n}$ & \multicolumn{1}{c}{$\%$} \\
\hline $0,00-$ & 0,075 & 0 & 0,00 & 0 & 0,00 \\
$0,075-$ & 0,75 & 16 & 66,67 & 12 & 60,00 \\
$0,75-$ & 1,87 & 8 & 33,33 & 7 & 35,00 \\
$1,87-$ & 3,75 & 0 & 0,00 & 1 & 5,00 \\
$3,75-$ & 7,50 & 0 & 0,00 & 0 & 0,00 \\
$7,50-15,00$ & 0 & 0,00 & 0 & 0,00 \\
\hline T O T A L & 24 & 100,00 & 20 & 100,00 \\
\hline
\end{tabular}


